\title{
CANCER
}

\section{Anti-CD73 slows tumour growth and spread}

Cancer cells are known to induce an immunosuppressive environment to evade host immune responses. The nucleoside adenosine, which often accumulates in tumours, has been identified as a player in this process by acting as a potent immunosuppressor. Now, Stagg and colleagues present a new strategy for breaking immune tolerance to tumour cells. They targeted CD73, an ectoenzyme involved in the generation of adenosine, and showed that the growth of breast tumours in a mouse model was significantly inhibited. Interestingly, targeting of CD73 also reduced chemotaxis of tumour cells and thereby the development of metastasis.

Cellular release of ATP in response to cell death or cellular stress is known to activate an immune response. By contrast, the hydrolysis of ATP to

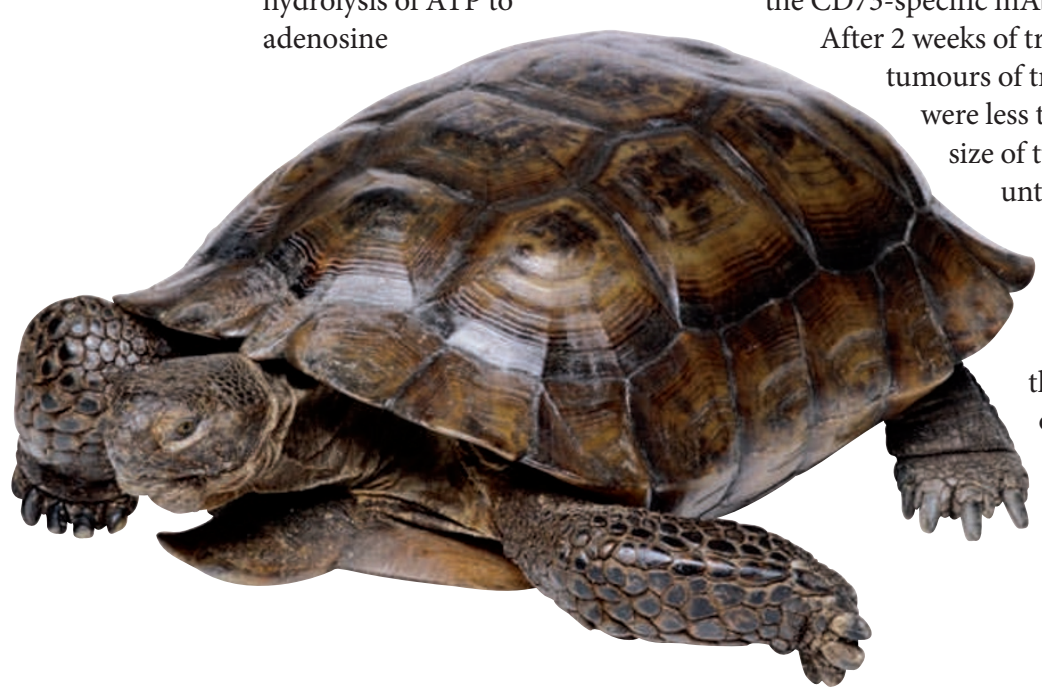

acts as a negative feedback mechanism and induces immunosuppression. A crucial component in this process is $\mathrm{CD} 73$, which catalyses the dephosphorylation of AMP to adenosine. CD73 is normally expressed on endothelial cells and subsets of haematopoietic cells, and is induced in response to cellular stress such as hypoxia. Importantly, it is overexpressed in a multitude of cancer cell types, including breast, prostate and bladder, as well as in melanoma, leukaemia and certain brain tumours.

To investigate CD73 as a target for anticancer strategies, the authors used a CD73-specific monoclonal antibody $(\mathrm{mAb})$ to treat mice injected with E0771 or 4T1.2 breast cancer cells. Starting 3 days after the inoculation with tumour cells, mice were intraperitoneally injected with
the CD73-specific mAb twice a week.

After 2 weeks of treatment, treated mice the untreated mice. Furthermore, the number of lung metastases in the spontaneously metastasizing $4 \mathrm{~T} 1.2$ breast cancer model was significantly reduced - even when comparing mice that had primary tumours of a similar size.

The experiments were repeated in severe combined immunodeficient mice. Results showed that inhibition of tumour growth due to treatment with a CD73-specific mAb was strictly dependent on an adaptive immune response, whereas the suppression of lung metastasis formation was not. Further molecular investigation identified the adenosine receptor $\mathrm{A}_{2 \mathrm{~B}}$ as the crucial mediator for adenosine-induced chemotaxis and metastasis of tumour cells.

This study provides proof-ofconcept for therapeutically targeting CD73. The authors point out that CD73-specific treatment might need to be combined with other forms of immune-stimulating therapies, as a patient's endogenous immune responses might be insufficient. Importantly, CD73 has also been implicated in the resistance to chemotherapeutic drugs such as doxorubicin, as well as resistance to apoptosis triggered by tumour necrosis factorrelated apoptosis-inducing ligand (TRAIL). This indicates that CD73specific agents could show synergistic effects with other anticancer drugs. Alexandra Flemming

ORIGINAL RESEARCH PAPER Stagg, J. et al. Anti-CD73 antibody therapy inhibits breast tumor growth and metastasis. Proc. Natl Acad. Sci. USA 4 Jan 2010 (doi: 10.1073/pnas. 0908801107) 